\title{
The Role of Space in Reducing Predator-Prey Cycles
}

\author{
Vincent A.A. Jansen and André M. de Roos
}

\subsection{Introduction}

Throughout the history of ecology, the interaction between predators and their prey has received attention from ecologists. Some of the longest and most well-known data series in ecology are from predator and prey populations, and predator-prey models are among the oldest in the field. Despite these efforts, a discrepancy exists between the behavior of most models and that of natural predator and prey populations. Most predator-prey models predict lasting periodic oscillations in population densities. Oscillations have been observed in nature, but they do not seem nearly as common or as pronounced as models predict.

Several explanations have been suggested for the qualitative difference between the behavior of the models and reality. Most of these involve additional mechanisms, such as invulnerable life stages of the prey or optimal foraging behavior of the predator. However, experimental studies show that the equations used in classical models do indeed give a reasonable qualitative description of the behavior of predator and prey populations (Gause et al. 1936; Maly 1969; Harrison 1995). All these studies compare model predictions with laboratory-scale experiments in which the densities often exhibit oscillations that can lead to population extinction (Huffaker 1958; Gause 1969; Luckinbill 1974). The models thus seem to describe the interaction between predators and their prey in laboratory experiments reasonably well, but fail to capture the properties of natural populations. Because there is no reason to believe that laboratory and natural populations are fundamentally different, the spatial scale at which the predator-prey system exists must play a crucial role in preventing population oscillations in the field. 
The spatial scale at which observations of ecological systems are carried out strongly influences their outcome. Hence, explanations for these observations should also take into account the spatial extent of the mechanisms considered. Modeling population dynamics should intuitively start at the level of the individual, at which the organisms reproduce, die, and interact, thus giving rise to population dynamics. At the individual level, it is appropriate to use a model that describes the whereabouts of all individuals and the interactions between them. However, describing the dynamics of the local or the global population in this manner is a daunting task. To unravel the dynamics at larger spatial scales, it would be preferable, or even necessary, to abstract individual-based descriptions of dynamics in terms of variables that are measurable at the scale of the local population. Subsequently, descriptions of the dynamics of the global population can be cast in terms of coupled local populations. The key issue is to find a non-phenomenological procedure to bridge the spatial scales - an issue that pervades many chapters in this book.

In this chapter, we describe models for three different spatial scales: those of the individual, the local population, and the global population. We first discuss some results from individual-based models. We then present a simple two-patch model for predator-prey interaction, followed by a generalization of this model to an arbitrarily large number of patches. Finally, we discuss how these results may be related to each other.

\subsection{Individual-based Predator-Prey Models}

For a spatially explicit description of interacting predator and prey individuals, one needs to keep track of all individuals and their positions. It will come as no surprise that these models are computationally complicated and intensive. Finding simplified models for such spatial, individual-based systems is an art in itself. Here, we try to make inferences directly from an individual-based, stochastic simulation model and argue that the emerging spatial dynamics reflect a system of coupled local populations where the local populations have a characteristic spatial extent. The dynamics of the (local) populations as observed at the characteristic spatial scale are close to the dynamics of the nonspatial, or homogeneously mixed, analogue of the spatial model. This naturally reduces the study of the global population dynamics to (1) finding the characteristic spatial scale of the local populations and (2) studying the dynamics of the coupled set of local populations.

The results we discuss are from "discrete-entity simulations" (de Roos et al. 1991; Wilson et al. 1993, 1995b; McCauley et al. 1993). These 
simulations describe a large lattice $(128 \times 128)$ on which predators and prey live. Every grid point can either be empty or occupied by at most one predator or one prey at a time. Hence, the whereabouts of every individual are known at every point in time. Predators and prey can move around independently in various ways. For instance, all individuals can be randomly redistributed over the entire lattice at every time step, resulting in homogeneous movement. Another possibility is diffusive movement in which the individuals randomly walk through the grid for a variable number of steps. The third option is a stationary population that can only occupy new space by growing into it. If a destination site is already occupied by an individual of the same species, movement does not take place. Once movement has taken place, a sequence of interactions follows: Prey individuals reproduce with a certain probability into a neighboring site. If no free site is available, the offspring is aborted. Similarly, predators die with a certain probability. If, as a result of individual movement, a prey and a predator end up at the same site, the predator eats the prey and subsequently reproduces with a certain conversion efficiency. The offspring is placed on the grid in the neighborhood of the parent. (For details of this simulation procedure see McCauley et al. 1993.)

Within this framework, the rules at the individual level can be varied to reflect different types of behavior for the predator and prey. We discuss some results from rules that have effects comparable to logistic prey growth and a type II functional response, given by the Holling disk equation (Holling 1965). This choice enables us to compare the behavior of this model with that of the classic nonspatial predator-prey model of Rosenzweig and MacArthur (1963), which is based on similar assumptions. In the simulations, prey growth is locally density dependent, because a prey offspring is aborted if the neighboring site where it attempts to establish itself is occupied. The type II functional response is mimicked by preventing the predators from eating for a number of time steps after they have consumed a prey. This results in a handling time for the predators (de Roos et al. 1991).

The simulation model behaves very much like the RosenzweigMacArthur ordinary differential equation (ODE) model when the prey is stationary and the predators move homogeneously. Both models have two qualitatively different types of dynamics: either the densities approach a stable equilibrium or they exhibit stable oscillations. Decreasing the predator death rate causes a transition from stable to oscillatory dynamics. Quantitatively, the transition occurs at similar values for the predator death rate in both the simulation and the ODE models (McCauley et al. 1993). 
In contrast, when the predators move diffusively and the prey remain stationary the simulation model behaves very differently: a decrease in the predator death rate has very little effect on the observed dynamics, and for all parameter combinations the densities deviate little from constant densities (de Roos et al. 1991; McCauley et al. 1993).

Why the difference? One possible explanation is that the classic ODE model does not accurately represent the interactions among the individuals when they move diffusively. For example, even though the rules for predator behavior in the simulations lead to a type II functional response at the population level when movement is homogeneous, diffusive movement might lead to a type III functional response when measured at the population level. It could be argued that the latter causes the observed stabilization of dynamics. However, as the observed functional responses in the simulation model with homogeneous or diffusive predator movement are very much like those in the classic model (de Roos et al. 1991), this explanation seems unlikely. A similar conclusion holds for the prey growth function. Hence, the explanation is probably not that the ingredients of the classic model are wrong, but that the spatial character of the interactions in the simulation model qualitatively changes the dynamics.

Spatial interactions can only influence the population dynamics if the population densities are not spatially homogeneous. Indeed, under diffusive predator movement the populations in the simulation model are clustered. Such clusters imply that, from an individual's point of view, the environment looks relatively homogeneous as long as it is perceived through a spatial window of about the size of the cluster. This locally experienced density might bear no relation to the overall densities as measured on large spatial scales. However, the dynamics at such local scales are similar to those predicted by the nonspatial model, where individuals mix homogeneously (de Roos et al. 1991). Figure 11.1 shows the coefficient of variation (the standard deviation divided by the average) of the time dynamics in prey density versus the size of the spatial window within which these dynamics are observed. For very small window sizes the coefficients of variations for diffusive movement and homogeneous movement are comparable; for larger window sizes the curves deviate. Also, the (temporal) autocorrelation function of these dynamics in prey density shows a convergence to the autocorrelation predicted by the nonspatial model when the observations on prey density are carried out at smaller spatial scales (de Roos et al. 1991).

The window size where the curves start to deviate can be designated as the characteristic spatial scale of the system. Measured at spatial scales much larger than this characteristic scale, the dynamics are the cumulative 


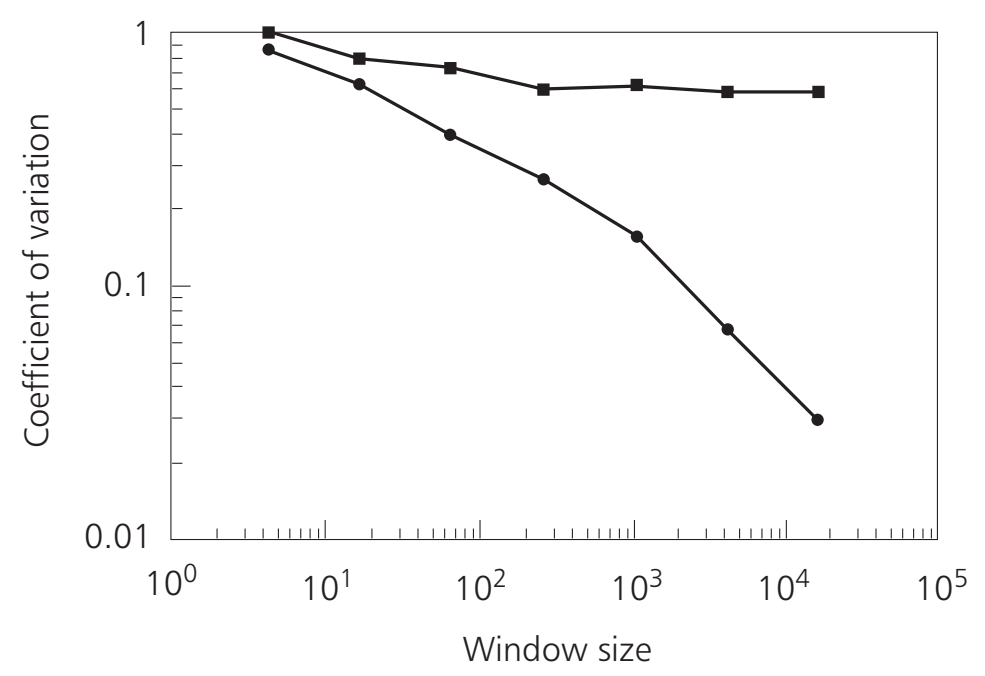

Figure 11.1 Variability in prey density in terms of the coefficient of variation of the discrete-entity simulations. Squares indicate homogeneous predator movement; circles indicate diffusive predator movement. Source: de Roos et al. (1991).

result of processes in only loosely coupled regions. At much smaller spatial scales, the dynamics are increasingly influenced by stochastic fluctuations due to the finite number of individuals present. At the characteristic spatial scale the system thus behaves in a more deterministic manner than at any other scale (Rand and Wilson 1995; see Chapter 12).

\subsection{A Deterministic Model of Two Coupled Local Populations}

We make the step from a description at the level of the individual to the level of the local population using the observation that at the characteristic spatial scale determinism is maximal and the populations behave as if they are well mixed. This justifies a description of local populations in terms of their mean densities only. To describe the populations beyond the characteristic spatial scale, we couple a number of local populations diffusively by assuming that every individual has a fixed probability of leaving its local population and migrating to another.

We investigate how fluctuations in the densities in the local populations affect the dynamics of the global population. Ideally, one would derive the equations that describe the local populations from the rules that govern the simulation model. Because it is still an open question how this should be done, we make life simple by boldly assuming that the local populations behave like standard predator-prey models. The simplest predator-prey model is the Lotka-Volterra model, and we start our investigations with the simplest spatial extension of this model, the two-patch Lotka-Volterra 
model (Comins and Blatt 1974). In terms of dimensionless variables, the model can be specified as

$$
\begin{aligned}
& \dot{N}_{1}=r N_{1}-N_{1} P_{1}, \\
& \dot{P}_{1}=N_{1} P_{1}-\mu P_{1}+\frac{m}{2}\left(P_{2}-P_{1}\right), \\
& \dot{N}_{2}=r N_{2}-N_{2} P_{2}, \\
& \dot{P}_{2}=N_{2} P_{2}-\mu P_{2}+\frac{m}{2}\left(P_{1}-P_{2}\right),
\end{aligned}
$$

where the dot means differentiation with respect to time. It is assumed that the local prey density, $N_{i}$, increases exponentially with rate $r$ in the absence of predators and that there is a linear contact rate between local prey and predators. The densities are scaled such that all prey mass that is eaten reappears as predator mass. In the absence of prey the local predator density, $P_{i}$, decreases exponentially with rate $\mu$. The two populations are coupled through migrating predators, which move to a neighboring patch at rate $\frac{m}{2}$. (The migration rate is divided by two because in the more general situation of a chain of connected patches in which every patch has two neighbors the migrants leave a patch at rate $m$. Because this system consists of only two patches, half the emigrants will be reflected from the system's boundary and the effective migration rate is $\frac{m}{2}$.) The prey are assumed to be stationary as in the discrete-entity simulations.

As long as the densities of the predator and the prey are the same in both patches (i.e., $P_{1}=P_{2}, N_{1}=N_{2}$, the system is completely homogeneous), the net effect of migration is zero and the model is identical to the nonspatial Lotka-Volterra model. The dynamics of the nonspatial model are well known: a family of closed orbits surrounds a neutrally stable equilibrium, and the densities oscillate eternally with an amplitude that depends on the initial conditions.

For Lotka-Volterra models in a spatially continuous domain the densities eventually become homogeneous (Murray 1975). The same holds for the two-patch Lotka-Volterra model: all differences in density between the patches disappear asymptotically for any positive initial condition. [This can be shown using the Lyapunov function $V_{1}(t)+V_{2}(t)$, where $V_{i}(t)=N_{i}+P_{i}-\mu \ln N_{i}-r \ln P_{i}$.] One therefore might not expect the dynamics of the spatial Lotka-Volterra model to yield any insights that cannot be gained from the nonspatial Lotka-Volterra model. Surprisingly, this is not the case. Figure 11.2 shows a solution of Equations (11.1) starting with small differences in densities between the patches. Although the differences between the prey and predator densities in the two patches eventually 
(a)

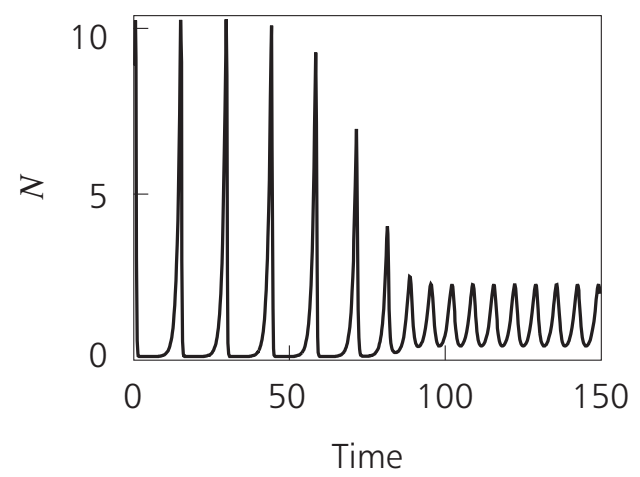

(b)

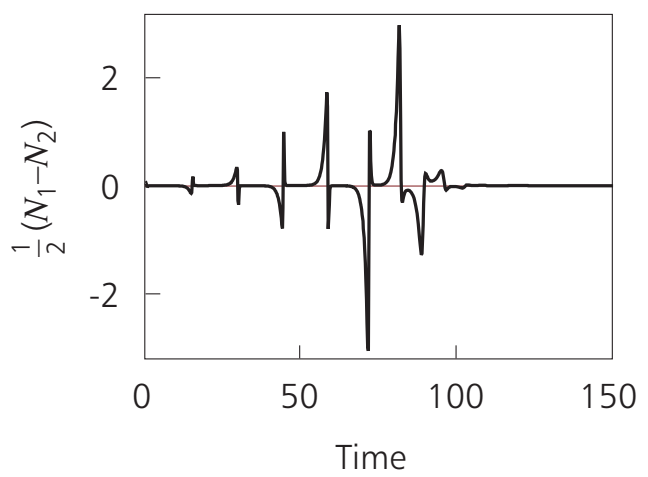

Figure 11.2 A solution of Equations (11.1) for $r=\mu=1$. (a) Average prey density $(N)$. (b) Differences between prey densities in the two patches $\left(\psi_{N}\right)$. The predator densities exhibit similar fluctuations (not shown).

disappear, they initially increase (see Figure 11.2b). As the differences increase, the amplitude of fluctuations in the average densities are reduced.

To understand the behavior of the model given by Equations (11.1), it helps to consider the geometry of the four-dimensional state space. The part of the state space in which there are no differences in densities between the patches - that is, $N_{1}=N_{2}$ and $P_{1}=P_{2}$ - is represented by an invariant two-dimensional plane. On this diagonal plane the dynamics are given by the (nonspatial) Lotka-Volterra model: closed orbits surround an equilibrium. The orbit shown in Figure 11.3 starts near a closed orbit in this plane, moves away from it, and then returns to another closed orbit in the diagonal plane on which the amplitude of the fluctuation is much smaller. Not all closed orbits in the diagonal plane have similar stability properties: some attract while others repel.

To establish which of the closed orbits attract and which repel, we analyze their (local) stability. To this end we introduce variables for the average prey and predator densities, $N=\frac{1}{2}\left(N_{1}+N_{2}\right), P=\frac{1}{2}\left(P_{1}+P_{2}\right)$, and a vector that contains the differences between the patches,

$$
\psi=\left(\begin{array}{l}
\psi_{N} \\
\psi_{P}
\end{array}\right)=\left(\begin{array}{c}
\frac{1}{2}\left(N_{1}-N_{2}\right) \\
\frac{1}{2}\left(P_{1}-P_{2}\right)
\end{array}\right) .
$$

In new variables, the system reads as follows:

$$
\begin{aligned}
\dot{N} & =r N-N P-\psi_{N} \psi_{P} \\
\dot{P} & =N P-\mu P+\psi_{N} \psi_{P} \\
\dot{\psi}_{N} & =(r-P) \psi_{N}-N \psi_{P} \\
\dot{\psi}_{P} & =P \psi_{N}+(N-\mu-m) \psi_{P} .
\end{aligned}
$$




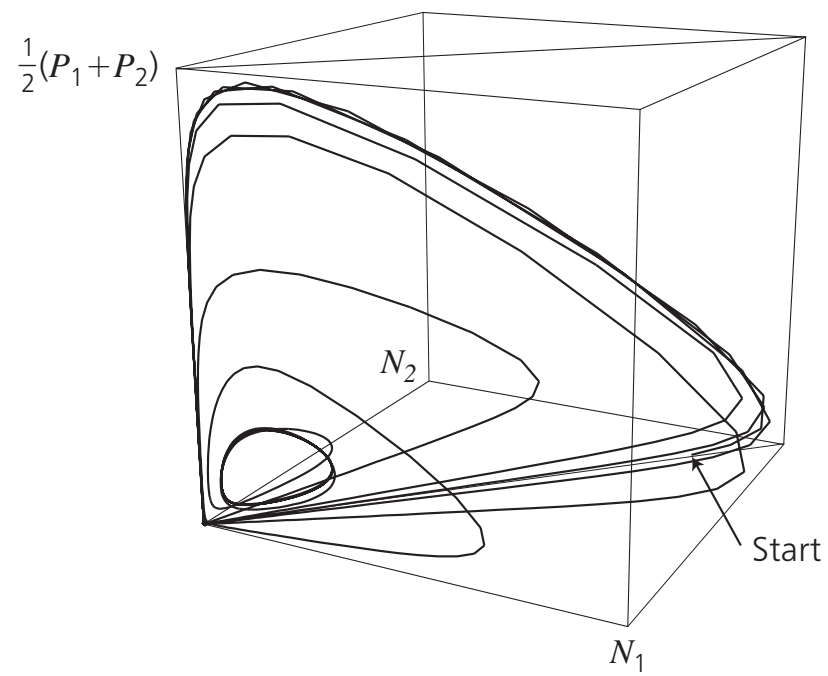

Figure 11.3 The solution visualized in Figure 11.2 in a projection in the $N_{1}, N_{2}, \frac{1}{2}\left(P_{1}+\right.$ $\left.P_{2}\right)$ space. The orbit starts at the arrow.

The dynamics of Equations (11.1) close to the diagonal plane are given by a linearization in the neighborhood of $|\psi|=0$ :

$$
\begin{aligned}
& \dot{N}=r N-N P \\
& \dot{P}=N P-\mu P,
\end{aligned}
$$

and

$$
\dot{\psi}=\left(\begin{array}{cc}
r-P & -N \\
P & N-\mu+\lambda m
\end{array}\right) \psi
$$

where the variable $\lambda$ is introduced for later use and here takes the value -1 . Notice that the system for the averages, Equations (11.4), is identical to the nonspatial Lotka-Volterra model. The linearized equations for the differences, Equation (11.5), are driven by the Jacobian of Equations (11.4) with an additional diagonal term representing migration.

The closed orbits in the diagonal plane are represented by solutions of Equations (11.4) and (11.5) with $\psi=0$. To see whether a closed orbit attracts or repels, we take an initial value for $N$ and $P$ and integrate Equations (11.4). Because Equations (11.4) are decoupled from Equation (11.5), we can take $\psi \neq 0$ without changing the solutions of Equations (11.4); in other words, in the linearization we can introduce differences between the patches while keeping the mean densities behaving as if there are no differences. A closed orbit repels if $|\psi|$ increases in the long run. A more formal, but essentially similar method of establishing the stability of the closed orbits is determining the dominant Floquet multiplier of Equation (11.5) (see Box 11.1). 


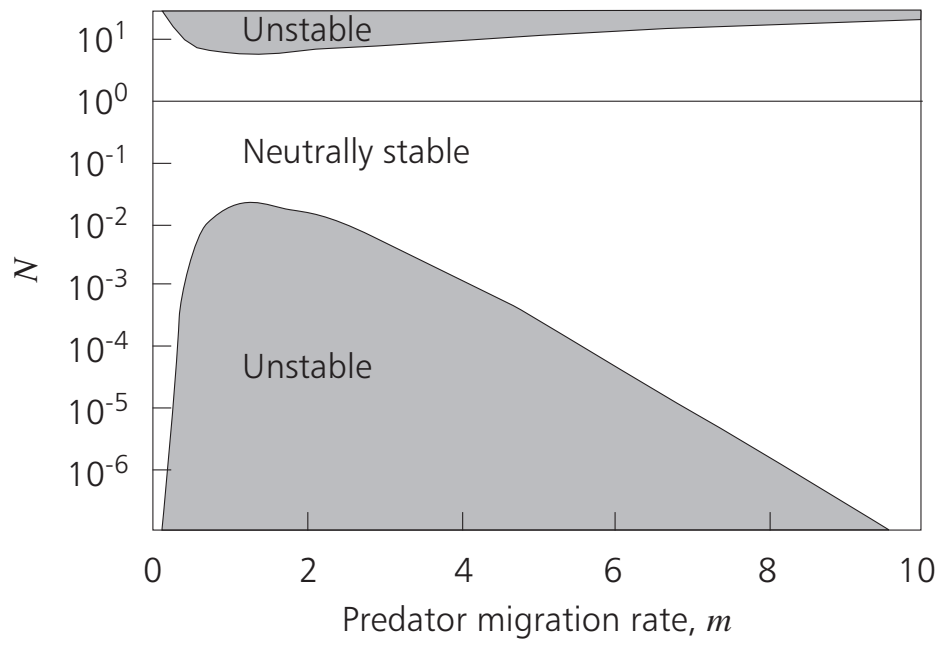

Figure 11.4 Minimum and maximum prey densities over the closed orbit of Equations (11.1) with a multiplier at -1 for different values of the predator migration rate. Closed orbits with a larger amplitude than the closed orbit with multiplier at -1 are unstable and repel; closed orbits with a smaller amplitude are neutrally stable and attract orbits over which the densities in the patches differ. Therefore, this graph also represents the maximum and minimum prey densities in the two-patch Lotka-Volterra system given by Equations (11.1) after transients have died out. Parameter values: $r=\mu=1$.

The closed orbits of the Lotka-Volterra model have two multipliers equal to 1 and hence are neutrally stable. Therefore, the closed orbits on the diagonal plane, described by Equations (11.4), also have two multipliers equal to 1 and two others, determined by Equation (11.5), that represent the spatial interactions. Because the dynamics on the diagonal are identical to the nonspatial dynamics, the spatial interactions can destabilize these orbits but can never stabilize them.

Figure 11.3 suggests that closed orbits on which the densities oscillate with a large amplitude can be unstable, while smaller closed orbits attract. Figure 11.4 shows that this is indeed the case for a large range of migration rates. The large closed orbits are unstable because the absolute value of one of the multipliers exceeds 1 (it is real, negative, and smaller than -1). For small closed orbits, the absolute value of the multipliers determined by Equation (11.5) is smaller than 1. These closed orbits attract orbits from outside the diagonal plane. (They are neutrally stable because they also have two multipliers at 1.) Between the neutrally stable and the unstable closed orbits lies a single closed orbit with a multiplier at -1 . Figure 11.4 shows the minimum and maximum prey values attained at this closed orbit.

The two-patch Lotka-Volterra model can thus behave very differently from its nonspatial counterpart. In the nonspatial model fluctuations of all amplitudes are possible. In contrast, in the spatial model homogeneous 
Box 11.1 Floquet multipliers

The stability of periodic solutions is established by considering a cross section to the periodic orbit, the Poincaré section (see illustration).
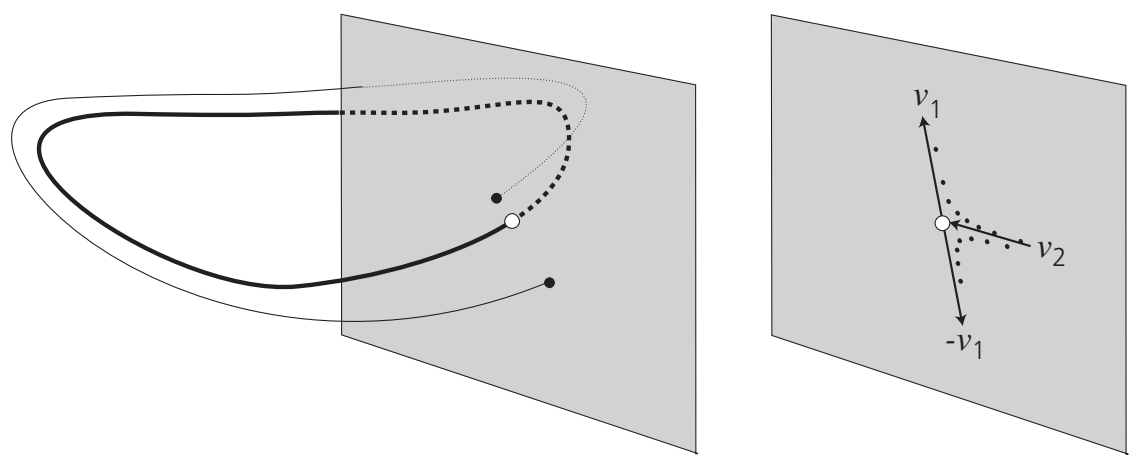

The Poincaré map is the next intersection of an orbit with a cross section to the periodic solution, the Poincaré section (left). The periodic orbit is a fixed point of the Poincaré map; it is unstable if nearby orbits move away from it (right). $v_{1}$ and $v_{2}$ are respectively the unstable and stable eigenvectors of the linearized Poincaré map $B(T)$.

Starting from the intersection point of the orbit with this plane, the system will obviously return to this same point in the state space every $T$ time units, where $T$ is the period of the solution. The periodic solution is stable if orbits that are close to it in the state space converge to it over time. Whether this occurs or not can be studied by analyzing a related discrete time system, the so-called Poincaré map, which maps the intersection point of a particular orbit with the Poincaré section to the intersection point after the next turn-around. The periodic solution is stable if the fixed point of the Poincaré map (which corresponds to the intersection point of the Poincaré section and the periodic orbit) is stable.

The stability of the discrete system can be determined from its linearization, but with a twist: to arrive at a computational procedure we linearize the underlying continuous time system in the neighborhood of the periodic orbit. The calculation is the same as for linearizing around an equilibrium, that is, we temporarily fix a point $x(t)$ on the periodic orbit and linearize the right-hand side of the differential equation around this point to arrive at

$$
\frac{d y}{d t}=F(t) y \text {, }
$$

where $y(t)$ denotes a small perturbation relative to $x(t)$, and $F(t)$ denotes the local matrix of partial derivatives of the right-hand side of our original differential equation for $x$, evaluated at the point $x(t)$. The only difference from the usual linearization around an equilibrium is that $F$ now depends on time in a $T$-periodic fashion due to the movement of $x$ around the periodic orbit, so that $F(t+T)=F(t)$. Periodic linear systems of differential equations such as (a) do not allow an explicit solution. But that does not matter as we only need numerical solutions from $t=0$ to $t=T$ for the special initial conditions continued 
Box 11.1 continued

$$
y(0)=\left(\begin{array}{c}
1 \\
0 \\
\vdots \\
0
\end{array}\right), \ldots, y(0)=\left(\begin{array}{c}
0 \\
\vdots \\
0 \\
1
\end{array}\right) \text {. }
$$

These are easily calculated. We glue the resulting columns $y(t)$ behind each other into a matrix $B(t) . B(t)$ indicates how the neighborhood of $x(0)$ is transformed into the neighborhood of $x(t)$ by the dynamical system acting over a time $t$. For the special value $t=T$

$$
y(T)=B(T) y(0),
$$

we get a map of the neighborhood of $x(0)$ onto itself. The eigenvalues $m_{i}$ of $B(T)$ are the so-called Floquet multipliers of the linear system. In the present context they are also referred to as the Floquet multipliers associated with the periodic orbit. For such special periodic systems $B(T)$ necessarily has one eigenvalue $m_{1}=1$ corresponding to an eigenvector pointing from $x(0)$ in the direction of the orbit; the other Floquet multipliers $m_{i}, i=2, \ldots$, are equal to the eigenvalues of the linearized Poincaré map (see, e.g., Hartman 1964). If all $\left|m_{i}\right|<1$ for $i=2, \ldots$, the periodic orbit is asymptotically stable. If at least one $\left|m_{i}\right|>1$, it is unstable.

large amplitude solutions can be diffusively unstable; consequently, in the long run fluctuations with a large amplitude will not be observed.

\subsection{Larger Spatial Domains}

The possibilities for oscillations in a two-patch Lotka-Volterra system are restricted because closed orbits with large amplitude can be diffusively unstable. The coupling between the two patches can bound the oscillations of the entire predator-prey system. Often, the spatial domain will be much larger than twice the characteristic spatial scale. Are the oscillations in densities of larger systems also reduced? If so, how do results from two-patch systems relate to systems with more patches? To answer these questions, we investigate the behavior of the equivalent of Equations (11.1) with $n$ patches:

$$
\begin{aligned}
& \dot{N}_{j}=r N_{j}-N_{j} P_{j} \\
& \dot{P}_{j}=N_{j} P_{j}-\mu P_{j}+m \sum_{i=1}^{n} c_{i j} P_{i} .
\end{aligned}
$$


Again, it is assumed that prey is stationary, hence the change in prey density in patch $j, N_{j}$, depends only on the local prey and predator densities. The predator density in patch $j, P_{j}$, changes through reproduction after prey consumption, through predator death (which depends only on local densities), and through migration of predators. The rate of predator emigration from patch $j$ is given by $m c_{j j}$. The predator density in patch $j$ changes through immigration from patch $i$ to $j$ at rate $m c_{i j}$ (note that the use of indices is contrary to the convention in the deterministic literature). The matrix $C=\left(c_{i j}\right)$ depends on the spatial organization and the size of the patches. Although our analysis below holds more generally, we only consider a linear chain of $n$ equal-sized, identical patches. In this case,

$$
C=\left(\begin{array}{ccccccc}
-\frac{1}{2} & \frac{1}{2} & 0 & \ldots & & \ldots & 0 \\
\frac{1}{2} & -1 & \frac{1}{2} & \ddots & & & \vdots \\
0 & \frac{1}{2} & -1 & \frac{1}{2} & \ddots & & \\
\vdots & \ddots & & & & \ddots & \vdots \\
& & \ddots & & & & 0 \\
\vdots & & & \ddots & \frac{1}{2} & -1 & \frac{1}{2} \\
0 & \ldots & & \ldots & 0 & \frac{1}{2} & -\frac{1}{2}
\end{array}\right) .
$$

The parameter $m$ hence represents the maximum emigration rate from a single patch.

We now analyze the stability of spatially homogeneous solutions, that is, solutions for which the densities in all patches are equal. Because we assume that all patches have the same size and their local dynamics are identical, the migration terms cancel when no spatial differences in the densities exist, and the dynamics of Equations (11.6) again reduce to those of the nonspatial Lotka-Volterra system. As with the two-patch Lotka-Volterra model, the model has a two-dimensional diagonal subspace in which closed orbits surround a neutrally stable equilibrium. Using the same method as for the two-patch model, it can be shown that solutions of Equations (11.6) with positive initial conditions converge to the diagonal: differences in densities disappear asymptotically.

To find out whether the possible range of fluctuations is reduced in the multi-patch model as in the two-patch model, the stability of the closed 
Box 11.2 Local stability analysis in multi-patch models

In a system of connected, identical patches, in the absence of migration the local dynamics in all patches can generally be described by

$$
\dot{x}=f(x)
$$

[ $x$ is a vector containing the $k$ different species' densities, $f(x): \mathbb{R}^{k} \rightarrow \mathbb{R}^{k}$ is a vector-valued function]. A homogeneous solution in such a multi-patch system would mean that the densities in all patches are the same and thus net migration is zero. Let $s(t)$ denote the time-course of all densities in a patch in the homogeneous solution, $s(t)$ necessarily is a solution of Equation (a). In Appendix 11.A it is shown that such a homogeneous solution is stable if, for all $i, \psi=0$ is an asymptotically stable equilibrium for

$$
\dot{\psi}=\left[D f(s(t))+\lambda_{i} M\right] \psi,
$$

and unstable if $\psi=0$ is an unstable equilibrium for at least one $i$. [Here $\lambda_{i}$ is an eigenvalue of the matrix $C$, which describes how the patches are connected, $M$ is a diagonal matrix with the species' migration rates on its diagonal, and $D f(s(t))$ is the Jacobian of $f$, evaluated at $s(t)$.]

This offers a generally applicable method to uncouple the local dynamics and reduce the complexity of spatial models.

orbits on the diagonal plane needs to be assessed. Although at first a stability analysis of a $2 \times n$ dimensional system might seem forbidding, it turns out to be simple: the problem can be reduced to $n$ decoupled twodimensional systems similar to Equation (11.5) (see Box 11.2). This enables us to relate the stability of the multi-patch model to that of the twopatch model by a scaling of the migration rate. The only information that is needed about the spatial structure are the eigenvalues of the connectivity matrix $C$. We now demonstrate the effect of the size of the spatial domain on the oscillations in the predator-prey model. The eigenvalues of the matrix $C$, given above, equal $\lambda_{i}=-1+\cos \frac{i}{n} \pi$, where $i=1, \ldots, n$. For a chain consisting of a single patch, there are no spatial interactions and hence no restrictions on the possible fluctuations. For a chain of two patches, there is one eigenvalue of $C$ different from zero with value -1 (the value of $\lambda$ used in the previous subsection). The maximum possible fluctuation can be read from Figure 11.4. Next consider a chain of three patches. The relevant eigenvalues are $-\frac{1}{2}$ and $-\frac{3}{2}$. To establish the size of possible fluctuations for a given $m$, we have to read the graph in Figure 11.4 at $\frac{1}{2} m$ and $\frac{3}{2} m$. Because closed orbits that are unstable for at least one of these two values will not be observed, only the most restrictive value matters. In 
(a)

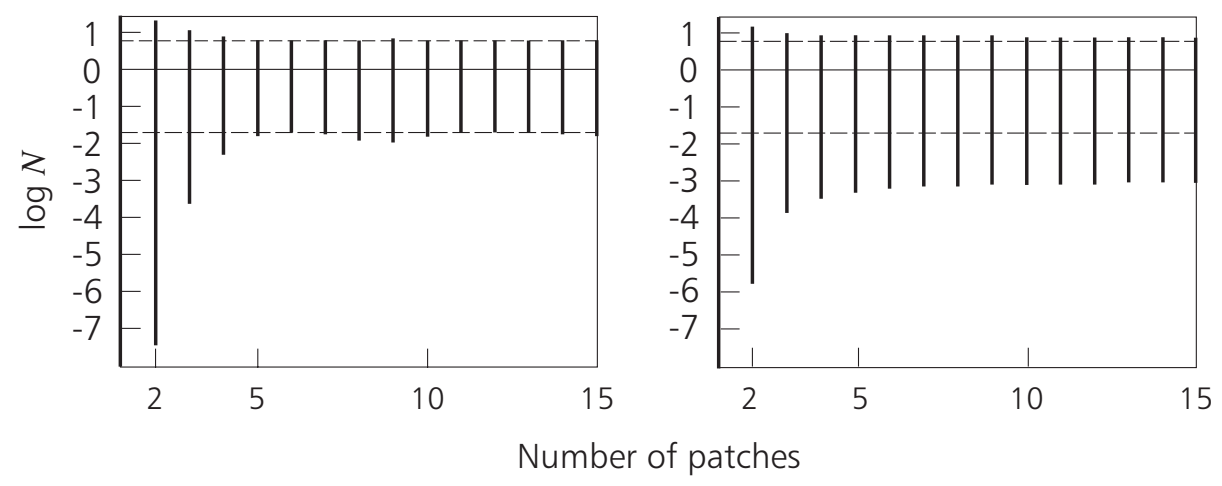

Figure 11.5 Maximum size of the neutrally stable closed orbits in a linear chain of patches versus chain length. This also represents the maximum observable oscillation after transients have died out. The dashed lines correspond to the extrema from Figure 11.4. Parameters: $r=\mu=1$; in (a) $m=10$, in (b) $m=0.2$.

this way we can construct a diagram of the maximum possible fluctuations versus the length of the chain (Figure 11.5). With increasing chain length, the number of eigenvalues increases and it becomes more likely that a value lies within the range of migration rates for which the oscillations are maximally reduced in Figure 11.4. The range of predator migration rates for which the fluctuations will be reduced therefore increases with the size of the spatial domain (the number of patches). For migration rates larger than that for which the curve in Figure 11.4 attains its minimum, the amplitude of the maximum observable fluctuations converges toward the minimum for a two-patch system, as can be seen in Figure 11.5a. For smaller migration rates the observable fluctuations converge with increasing domain size toward either the minimum value possible or a larger value (Figure 11.5b). Because for a linear chain of patches the eigenvalues of $C$ lie between 0 and -2 , this value is the maximum fluctuation possible for twice the migration rate in the two-patch case.

\subsection{The Spatial Rosenzweig-MacArthur Model}

The solutions of the multi-patch Lotka-Volterra model become spatially homogeneous, which prevents statistical stabilization as it occurs in the discrete-entity simulations. This is not a general property of multi-patch models. The Lotka-Volterra models are useful for obtaining some detailed insights into spatial predator-prey systems, but the well-mixed counterparts to the discrete-entity simulations are not of the Lotka-Volterra type but of the more general Rosenzweig-MacArthur type. Therefore, we briefly discuss our present understanding of the spatial Rosenzweig-MacArthur model, starting with a two-patch version (Jansen 1994, 1995). 
When the equilibrium of the nonspatial Rosenzweig-MacArthur model is unstable a stable limit cycle exists. The two-patch version of this model has a spatially homogeneous limit cycle that can be unstable for intermediate predator migration rates. This spatially homogeneous limit cycle is stable when the predator migration rate in a two-patch model is low. Beyond a critical value of predator migration, the limit cycle is unstable, as are the larger neutrally closed orbits of the two-patch Lotka-Volterra model. In contrast to the Lotka-Volterra model, the differences between the patches in the spatial Rosenzweig-MacArthur model persist, giving rise to qualitatively different dynamics. With predator migration slightly larger than the critical value, the dynamics are intermittent: most of the time they exhibit what appears to be a regular oscillation that is interrupted from time to time by "bursts" of irregular behavior (Bergé et al. 1984). In the twopatch Rosenzweig-MacArthur model the nearly regular oscillation appears when orbits dwell in the neighborhood of the unstable spatially homogeneous limit cycle. The bursts of irregular behavior occur when orbits leave the vicinity of the unstable homogeneous limit cycle in a fashion similar to that shown in Figure 11.2. For a short time the densities in the patches differ greatly and the amplitude of the oscillation is reduced. Then the differences in density decrease again and, slowly, the amplitude of the oscillation increases as the orbit reapproaches the unstable homogeneous limit cycle. For migration rates just over the critical value, this sequence of events is repeated in a chaotic fashion (type III intermittency), for larger migration rates it is repeated regularly and the dynamics are quasi-periodic (Jansen 1994).

In larger spatial domains the stability of the multi-patch RosenzweigMacArthur model can be calculated using the method described in Box 11.2. In the two-patch model the homogeneous limit cycle can be unstable for a relatively small range of intermediate predator migration rates. This range increases roughly with the square of the number of patches. In larger spatial domains the homogeneous limit cycle is stable only for very low or extremely high values of the predator migration rate. The homogeneous limit cycle loses its stability similarly to the two-patch model: for predator migration rates exceeding a critical value the dynamics become intermittent and a spatio-temporal pattern develops. The pattern is formed by groups of neighboring patches that oscillate almost in synchrony and are separated by regions in which the oscillations are reduced (Figure 11.6).

This behavior is comparable to the behavior of coupled map lattices (spatio-temporal intermittency, Kaneko 1993) and forms a transition between an ordered pattern and fully developed spatio-temporal chaos. The 
(a)

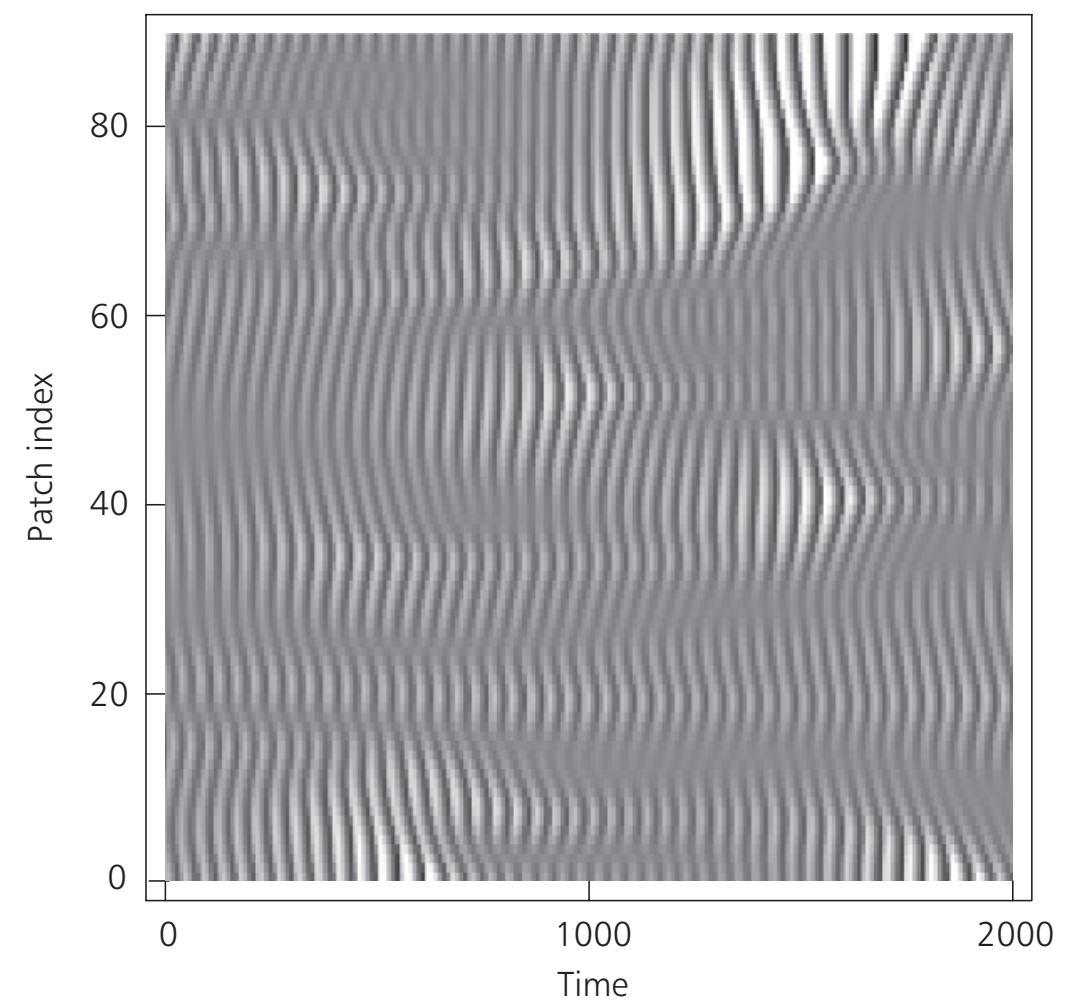

(b)

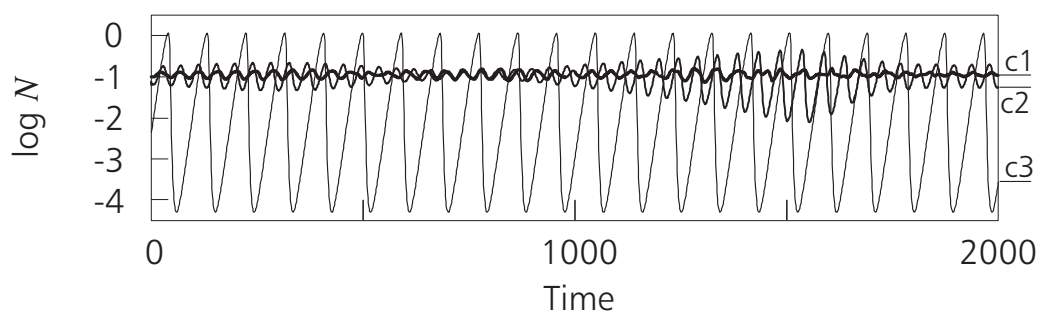

Figure 11.6 Solutions of a 90-patch Rosenzweig-MacArthur model, given by the equations $\dot{N}_{j}=N_{j}\left(1-N_{j} / 2\right)-b P_{j} N_{j} /\left(1+N_{j}\right), \dot{P}_{j}=b P_{j} N_{j} /\left(1+N_{j}\right)-P_{j}+m \sum_{i=1}^{n} c_{i j} P_{i}$. The patches are arranged in a linear chain with reflecting boundaries; the $c_{i j}$ are given in the text; $b=9.96$. For these parameters the homogeneous limit cycle is unstable for $0.2736<m<1832$; in this simulation $m=0.28$. (a) Prey densities in space and time. The darker the shading, the higher the prey densities. (b) Prey densities versus time, averaged over all patches (c1), in patch 40 (c2), and in the nonspatial model (c3).

phases of separated groups of synchronized patches are not related to each other. Therefore, the oscillations in the spatially averaged densities are reduced for two reasons: (1) because of the reduction in amplitude of the oscillations in the transition regions between groups of synchronized patches, and (2) because of the averaging combined with the phase differences between the synchronized groups of patches. In large spatial domains the total densities become nearly constant, again by statistical stabilization. 


\subsection{Concluding Comments}

We now discuss the relation between the results of the discrete-entity simulations and the simple multi-patch models. We claim that in both models the occurrence of large amplitude oscillations is prevented by statistical stabilization. However, the discrete-entity and multi-patch models do differ in some important aspects. First, discrete-entity simulations account for all spatial scales, from the individual up to the system size, whereas the multi-patch models only distinguish scales larger than the local-population scale. Second, demographic stochasticity is an inherent part of models that explicitly account for individual organisms, such as the discrete-entity simulations. In contrast, all processes in the multi-patch models are fully deterministic and phrased in terms of densities. Third, in the discrete-entity simulations movement is accounted for at the individual level and is fundamental for both the interactions among individuals (e.g., predation events) and the dispersal of individuals between different parts of the system. The multi-patch models only account for the dispersal of individuals between different parts of the system as a diffusion process, while mixing at the scale of the local population, which forms the basis of individual interactions, is assumed to be rapid and homogeneous.

We have indicated how the limited mobility in discrete-entity simulations naturally leads to a subdivision of the spatial scales into two categories: those that are larger than the characteristic spatial scale and those that are smaller than it. Therefore, the spatial structure in the discrete-entity simulations is somewhat akin to the structure artificially imposed by the multi-patch models. In addition, the prey growth rates and functional responses in regions smaller than the characteristic scale are similar to those in the simulations with homogeneous movement, which in large systems should be similar to Rosenzweig-MacArthur-type differential equations.

This view is based on the models we have studied so far and certainly needs to be substantiated more rigorously. However, if it is true, only two differences remain between the discrete-entity simulations and the multipatch models: (1) both the local dynamics and individual movement in the discrete-entity simulations are strongly influenced by demographic stochasticity, and (2) the purely diffusive movement of individuals in the discreteentity simulations need not necessarily lead to purely diffusive movement between local populations.

The question of whether the stabilizing mechanism in the two model frameworks is the same cannot be answered unambiguously with the information currently available. In our opinion, the key issue is to resolve 
the role of demographic stochasticity in the discrete-entity simulations. A speculative answer would be that the mechanisms are the same in the sense that both can be considered statistical stabilization, but that the causes of the effective uncoupling of the populations over the characteristic scale are of a different nature. In the multi-patch model, the uncoupling is due to deterministic forces that cause symmetry breaking and to the chaotic nature of the attractor. In the discrete-entity simulations, it is primarily a result of the independence of the individual stochastic events. These two mechanisms are not mutually exclusive but complement and reinforce each other. For parameter values for which symmetry breaking does not occur, the effect of demographic stochasticity may still be reinforced by the deterministic trend. This makes statistical stabilization a robust phenomenon that can explain many of the differences in dynamic behavior between spatial and nonspatial predator-prey systems.

Acknowledgments We thank Howard Wilson, John Prendergast, Jason Matthiopoulos, and John Lawton for comments on the manuscript.

\section{Appendix 11.A Stability Analysis of a Multi-patch System}

Consider a system of $n$ patches in which $k$ species live. The density of the $j$ th species in patch $i$ is described by $x_{i, j}$. The densities of all $k$ species are given by the vector $x_{i}=\left(x_{i, 1}, \ldots, x_{i, k}\right)^{T}$. To keep track of the densities of all species in all patches, we describe the state of the whole system with a $k \times n$ matrix $X=\left(x_{1}, \ldots, x_{n}\right)$ that has the densities of a particular species as rows and the densities of the $k$ species in a particular patch as columns. We assume that, from the perspectives of the interacting species, all patches are identical environments and that the local dynamics are defined by Equation (11.10). The global dynamics of the spatial system are given by a combination of local interactions and dispersal:

$$
\dot{X}=F(X)+M X C,
$$

where $F(X)=\left(f\left(x_{1}\right), \ldots, f\left(x_{n}\right)\right)$. The matrix $M$ is a $k \times k$ diagonal matrix that has the migration rates of the species on its diagonal. The matrix $C$ is an $n \times n$ matrix that describes how patches are connected in the system.

We perform a local stability analysis for solutions of Equation (11.8) that are spatially homogeneous, that is, for which for all $i ; x_{i}(t)=s(t)$, where $s(t)$ is a solution of Equation (a) in Box 11.2. Such flat solutions, which are denoted by $S(t)=(s(t), \ldots, s(t))$, exist when $C$ has a left eigenvector $(1, \ldots, 1)$ with eigenvalue 0 .

We transform $X(t)-S(t)$ into $\Psi(t)$ using the linear transformation $\Psi=(X-$ $S) A$, where $A$ is an $n \times n$ matrix that is invertible, hence $X-S=\Psi A^{-1}$. The 
time derivative of $\Psi$ is given by

$$
\begin{aligned}
\dot{\Psi} & =(\dot{X}-\dot{S}) A \\
& =(F(X)-F(S)) A+M(X-S) C A \\
& =(F(X)-F(S)) A+M \Psi A^{-1} C A .
\end{aligned}
$$

Matrix $A$ is chosen such that $A^{-1} C A=\Lambda$ is diagonal. This can be done if all eigenvectors of $C$ are different by choosing $A=\left(w_{1}, \ldots, w_{n}\right)$, where $w_{i}$ is a right eigenvector of $C$ - that is, $C w_{i}=\lambda_{i} w_{i}$ - and by choosing $A^{-1}=\left(v_{1}, \ldots, v_{n}\right)^{T}$, where $v_{i}$ is a left eigenvector of $C$ - that is, $v_{i} C=\lambda_{i} v_{i}$. The matrix $\Lambda$ has the eigenvalues $\lambda_{i}$ of $C$ on its diagonal. We choose $v_{1}=(1, \ldots, 1)$, which is a left eigenvector of $C$ with $\lambda_{1}=0$. Next we linearize the system around the homogeneous solution $S$ :

$$
\begin{aligned}
\dot{\Psi} & =(F(X)-F(S)) A+M \Psi \Lambda \\
& =D f(s)(X-S) A+M \Psi \Lambda+\text { h.o.t. } \\
& \approx D f(s) \Psi+M \Psi \Lambda .
\end{aligned}
$$

Thus $A$ transforms Equation (11.8) in the neighborhood of the spatially homogeneous solution in a system of $n$ decoupled subsystems, given by Equation (b) in Box 11.2. Hence, a spatially homogeneous solution of Equation (11.8) is asymptotically stable when $\psi_{i}=0$ is an asymptotically stable solution of Equation (b) in Box 11.2 for all $i$. Note that since the subsystem for $i=1$ is the linearization of Equation (a) in Box 11.2 around $s(t)$, a homogeneous solution $S$ can only be asymptotically stable if $s(t)$ is an asymptotically stable solution of Equation (a) in Box 11.2.

In general there are no methods to determine the stability of Equation (b) in Box 11.2 directly from Equation (a). However, the method described here can greatly reduce the numerical effort, because it permits extrapolating results from one spatial system (the simplest being a two-patch system) to more complicated systems. 


\section{References}

References in the book in which this chapter is published are integrated in a single list, which appears on pp. 517-552. For the purpose of this reprint, references cited in the chapter have been assembled below.

Bergé P, Pomeau Y \& Vidal C (1984). Order within Chaos. Chichester, UK: Wiley Comins HN \& Blatt DWE (1974). Prey-predator models in spatially heterogeneous environments. Journal of Theoretical Biology 48:75-83

de Roos AM, McCauley E \& Wilson WG (1991). Mobility versus density-limited predator-prey dynamics of different spatial scales. Proceedings of the Royal Society of London B 246:117-122

Gause GF (1969). Reprint. The Struggle for Existence. New York: Hafner Publishing Company. Original edition, Williams and Wilkins (1934)

Gause GF, Smaragdova P \& Witt AA (1936). Further studies of interaction between predators and prey. Journal of Animal Ecology 5:1-18

Harrison GW (1995). Comparing predator-prey models to Luckinbill's experiment with Paramecium and Didinium. Ecology 76:357-374

Hartman P (1964). Ordinary Differential Equations. New York, NY, USA: Wiley

Holling CS (1965). The functional response of predators to prey density and its role in mimicry and population regulation. Memoirs of the Entomological Society of Canada 45:5-60

Huffaker CB (1958). Experimental studies on predation: Dispersion factors and predator-prey oscillations. Hilgardia 27:343-383

Jansen VAA (1994). On the bifurcation structure of two diffusively coupled, predator-prey systems. In Theoretical Aspects of Metapopulation Dynamics, PhD dissertation, pp. 93-115, Leiden University, Leiden, Netherlands

Jansen VAA (1995). Regulation of predator-prey systems through spatial interactions: A possible solution to the paradox of enrichment. Oikos 74:384-390

Kaneko K (1993). Theory and Applications of Coupled Map Lattices. Chichester, UK: Wiley

Luckinbill L (1974). The effects of space and enrichment on a predator prey system. Ecology 55:1142-1147

Maly EJ (1969). A laboratory study of the interaction between the predatory rotifer Asplancha and Paramecium. Ecology 50:59-73

McCauley E, Wilson WG \& de Roos AM (1993). Dynamics of age-structured and spatially structured predator-prey interactions: Individual based models and population level formations. The American Naturalist 142:412-442

Murray J (1975). Non-existence of wave solutions for the class of reactiondiffusion equations given by the Lotka-Volterra equations with diffusion. Journal of Theoretical Biology 52:459-469

Rand DA \& Wilson HB (1995). Using spatio-temporal chaos and intermediatescale determinism to quantify spatially extended ecosystems. Proceedings of the Royal Society of London B 259:111-117

Rosenzweig ML \& MacArthur RH (1963). Graphical representation and stability conditions of predator-prey interactions. The American Naturalist 97:209-223 
Wilson W, de Roos A \& McCauley E (1993). Spatial instabilities within the diffusive Lotka-Volterra system: Individual-based simulation results. Theoretical Population Biology 43:91-127

Wilson W, McCauley E \& de Roos A (1995b). Effect of dimensionality on LotkaVolterra predator-prey dynamics: Individual based simulation results. Bulletin of Mathematical Biology 57:507-526 